Supporting Information

for

\title{
Effective Lorenz Number of the Point Contact between Silver Nanowires
}

\author{
Yucheng Xiong, ${ }^{\dagger, \perp}$ Yang Zhao, ${ }^{\ddagger}, \perp$ Yi Tao, ${ }^{\ddagger}, \S$ Fengju Yao, ${ }^{\dagger}$ Deyu Li, ${ }^{*, \star}$ and Dongyan $\mathrm{Xu}^{*, \dagger}$
}

${ }^{\dagger}$ Department of Mechanical and Automation Engineering, The Chinese University of Hong Kong, Shatin, New Territories, Hong Kong Special Administrative Region, China

\$Department of Mechanical Engineering, Vanderbilt University, Nashville, Tennessee 37235, United States

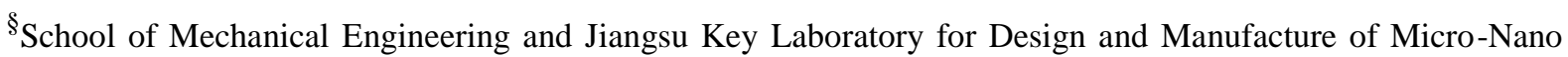
Biomedical Instruments, Southeast University, Nanjing 210096, China

\section{Corresponding Authors}

*E-mail: deyu.li@vanderbilt.edu.

*E-mail: dyxu@mae.cuhk.edu.hk. 


\section{Materials}

The Ag nanowires were purchased from XFNANO and Sigma-Aldrich and used as received. They were stored in isopropyl alcohol. The Ag nanowires measured in this work are penta-twinned nanowires with a pentagonal cross-section. We have confirmed this through directly imaging the cross section of the Ag nanowires of various sizes. As shown in Fig. S1a, all nanowires exhibit a pentagonal cross-section. The crystalline structure of the as-received Ag nanowires was characterized by HRTEM as shown in Fig. 1a, while the chemical composition was examined by HRTEM/energy dispersive X-ray spectrometry (HRTEM/EDX). The HRTEM/EDX spectrum of a representative sample is shown in Fig. S1b.

Before experiments, a selected Ag nanowire of tens of microns in length was cut into several segments using a home-made probe. These segments were then transferred onto the measurement microdevices to form the point-contact and single-nanowire samples. Fig. S1c and S1d show the SEM images of the contact between the Ag nanowire and the Pt electrode for the point-contact sample in Fig. 1b. As shown in S1a, the Ag nanowires studied in this work have a pentagonal cross-section. A sharp edge was observed for each segment in Fig. 1c, S1c, and S1d, as highlighted by the dashed lines, indicating that the wire preferentially lies on the Pt electrode with one side face, which renders the top edge of the bottom wire in contact with the bottom face of the top wire. This edge-face configuration is consistent with the SEM image in Fig. 1d. 
a

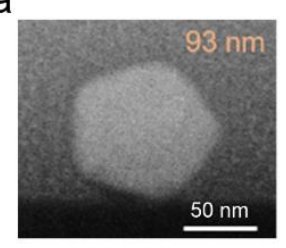

b

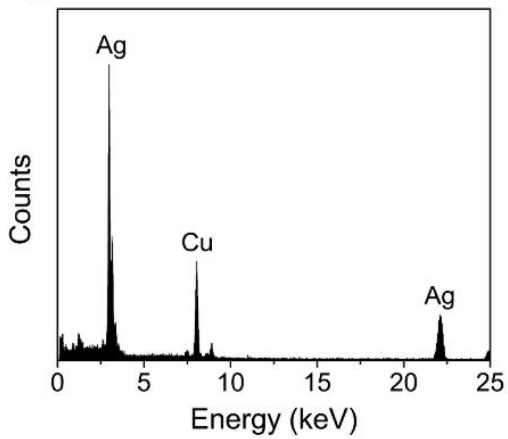

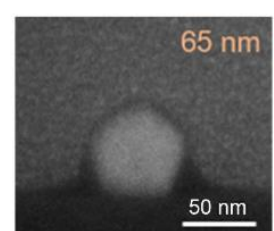

C

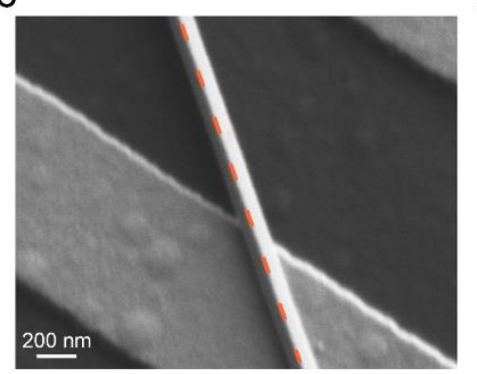

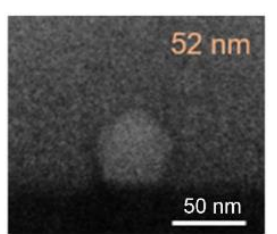

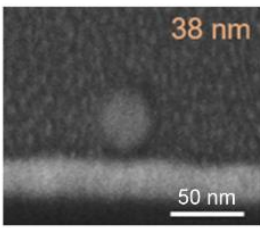

d

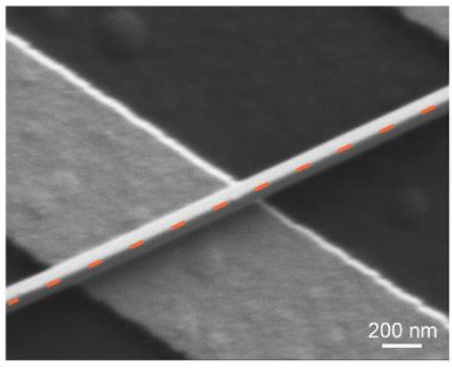

Figure S1. (a) SEM images of the cross section of the Ag nanowires of various sizes, with all nanowires exhibiting a pentagonal shape. (b) HRTEM/EDX spectrum of an Ag nanowire. The $\mathrm{Cu}$ signal comes from the TEM grid. (c, d) SEM images of the contact between the Ag nanowire and the Pt electrode for the point-contact sample in Fig. $1 \mathrm{~b}$.

\section{Thermal and electrical measurements}

The thermal and electrical resistance of the samples were measured over a temperature range from $20 \mathrm{~K}$ to $300 \mathrm{~K}$ by using a micro-thermal bridge method and a four-probe method, respectively. ${ }^{1,2}$ As shown in Fig. 1b, the measurement microdevice consists of two suspended membranes. Serpentine $\mathrm{Pt}$ coils were patterned on these membranes, serving as heaters/resistance thermometers for thermal measurement. Two separate Pt electrodes were also fabricated on each membrane, which enables the four-probe measurement. The electrical resistance was measured by passing an electrical current of less than $4 \mu \mathrm{A}$ through the sample to ensure that the Joule heating effect is minimal. To eliminate the convective heat loss, all measurements were performed in a cryostat system (Janis CCS-400H/204) with a high vacuum $\left(<10^{-6}\right.$ Torr). The suspended length and outer diameter of the measured Ag nanowires were characterized by SEM and TEM, respectively. 
To determine the effective Lorenz number of the point contact between Ag nanowires, we conducted a series of experiments on the point-contact and single-nanowire samples. Prior to the measurement, it is essential to ensure that $\mathrm{Ag}$ nanowires make good contact with $\mathrm{Pt}$ electrodes. To achieve this, an electrical current of less than $100 \mu \mathrm{A}$ was applied between the two Pt electrodes on each membrane. Previous studies ${ }^{3,4}$ have shown that the electrical treatment can lead to stable and low contact resistance between the Ag nanowire and the electrode. The measured total electrical and thermal resistance of the single-nanowire sample and the point-contact sample can be described as,

$$
\begin{aligned}
& R_{\text {tot_ } \_ \text {, } \mathrm{E}}=\frac{l_{\mathrm{S}}}{\sigma A}, R_{\mathrm{tot} \_\mathrm{S}, \mathrm{T}}=R_{\mathrm{wM}, \mathrm{T}}+\frac{l_{\mathrm{S}}}{\kappa A}, \\
& R_{\text {tot_C,E}}=\frac{l_{\mathrm{C}}}{\sigma A}+R_{\mathrm{C}, \mathrm{E}}, R_{\mathrm{tot} \_\mathrm{C}, \mathrm{T}}=R_{\mathrm{WM}, \mathrm{T}}+\frac{l_{\mathrm{C}}}{\kappa A}+R_{\mathrm{C}, \mathrm{T}},
\end{aligned}
$$

where subscripts tot, E, T, S, and C indicate the total electrical/thermal resistance, the electrical resistance, the thermal resistance, the single-nanowire sample, and the point-contact sample, respectively. $l$ and $A$ are the suspended length and cross-sectional area of the sample. $R_{\mathrm{C}, \mathrm{E}}$ and $R_{\mathrm{C}, \mathrm{T}}$ are the electrical and thermal resistance of the point contact between Ag nanowires. $R_{\mathrm{WM}, \mathrm{T}}$ is the contact thermal resistance between the wire and the two membranes, which is assumed to be constant among multiple measurements for the same Ag nanowire. The electrical conductivity was derived from the measured electrical resistance of the single Ag nanowires. The electrical conductivity extracted from the three single-nanowire segments for S1 essentially overlaps with each other (Fig. S2e), which confirms that the four-probe method effectively eliminates the effect of the contact electrical resistance between the wire and the suspended membranes. Through linear fitting of the measured thermal resistance $\left(R_{\text {tot_S,T }}\right)$ as a function of the suspended length $\left(l_{\mathrm{S}}\right)$ for the three single nanowires (Fig. S2f), $\kappa$ and $R_{\mathrm{WM}, \mathrm{T}}$ can be simultaneously extracted. Then, $R_{\mathrm{C}, \mathrm{E}}$ and $R_{\mathrm{C}, \mathrm{T}}$ of the point contact can be calculated 
according to Eq. (S2). The effective Lorenz number of the point contact is determined subsequently.

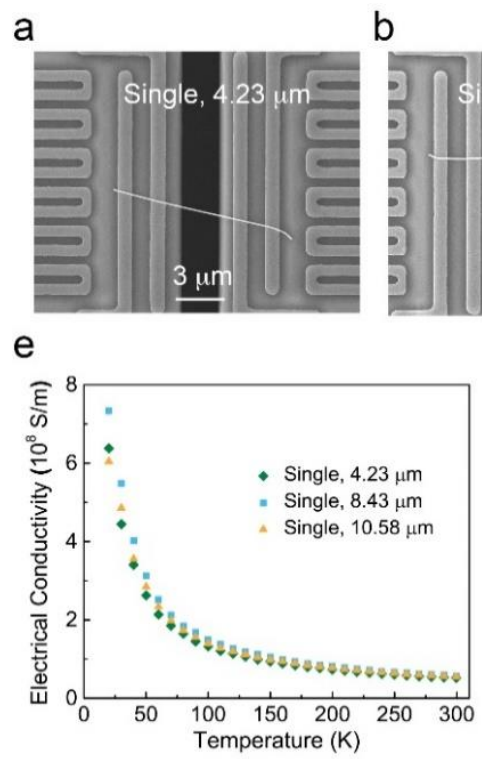

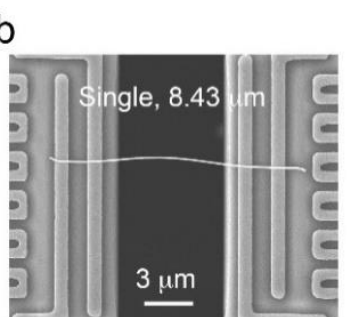

$f$

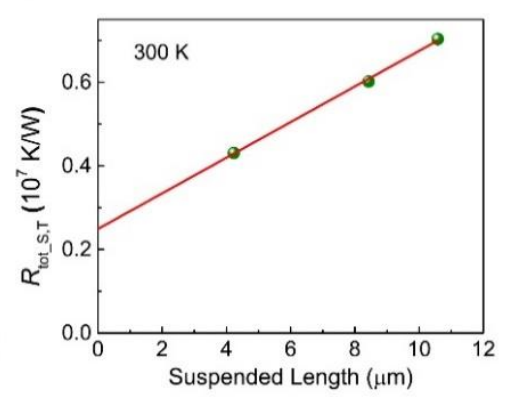

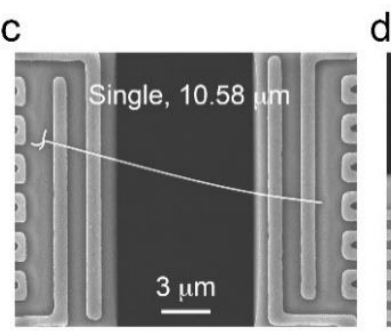

g

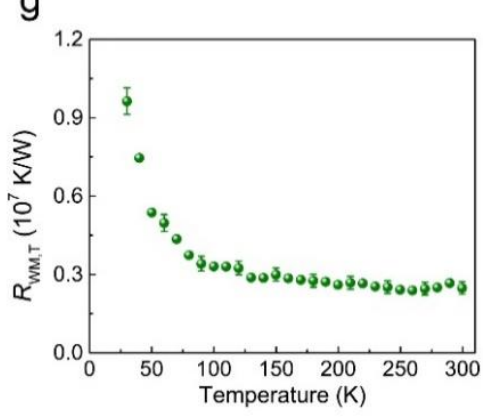

Figure S2. SEM images of (a-c) three single Ag nanowires with different suspended lengths and (d) two Ag nanowires with a point contact for S1. (e) Electrical conductivity extracted from the three single Ag nanowires nearly overlaps with each other, confirming that the contact electrical resistance is negligible. (f) Linear fitting of the measured thermal resistance $\left(R_{\text {tot_S,T }}\right)$ as a function of the suspended length $\left(l_{\mathrm{S}}\right)$ for the three single $\mathrm{Ag}$ nanowires at $300 \mathrm{~K} .(\mathrm{g})$ Extracted contact thermal resistance $\left(R_{\mathrm{WM}, \mathrm{T}}\right)$ between the wire and the two membranes. The hydraulic diameter of $\mathrm{S} 1$ is $92 \mathrm{~nm}$.

The assumption that $R_{\mathrm{WM}, \mathrm{T}}$ is constant among different measurements for the same $\mathrm{Ag}$ nanowire is verified by the linear relationship between $R_{\mathrm{tot} \_\mathrm{S}, \mathrm{T}}$ and $l_{\mathrm{S}}$, as shown in Fig. S2f. An alternative approach is to carefully adjust the suspended lengths of the single-nanowire sample and the point-contact sample to be nearly identical to each other. Doing so, $R_{\mathrm{C}, \mathrm{E}}$ and $R_{\mathrm{C}, \mathrm{T}}$ of the point contact can be simply obtained by $R_{\mathrm{C}, \mathrm{E}}=R_{\mathrm{tot} \_\mathrm{C}, \mathrm{E}}-R_{\mathrm{tot} \_\mathrm{S}, \mathrm{E}} \times l_{\mathrm{C}} / l_{\mathrm{S}}$ and $R_{\mathrm{C}, \mathrm{T}}=R_{\mathrm{tot} \_\mathrm{C}, \mathrm{T}}-R_{\mathrm{tot} \_\mathrm{S}, \mathrm{T}} \times l_{\mathrm{C}} / l_{\mathrm{S}}$, respectively. ${ }^{5}$ In addition, we applied a small drop of alcohol on 
each membrane to improve the contact between the Ag nanowire and the Pt electrodes as alcohol evaporation can pull the wire and the membrane together tightly. EBID of Pt/C on each electrode was also done to further improve the contact for S4. In fact, the thermal conductance of the Ag nanowire measured after the first and second rounds of EBID overlaps with each other, as shown in Fig. $\mathrm{S} 3 \mathrm{c}$, indicating that $R_{\mathrm{WM}, \mathrm{T}}$ is negligible after the EBID treatment. The consistent results from all these different contact treatments indicate that we have been able to minimize the contact thermal resistance between the nanowire and the suspended membranes to a negligible level.
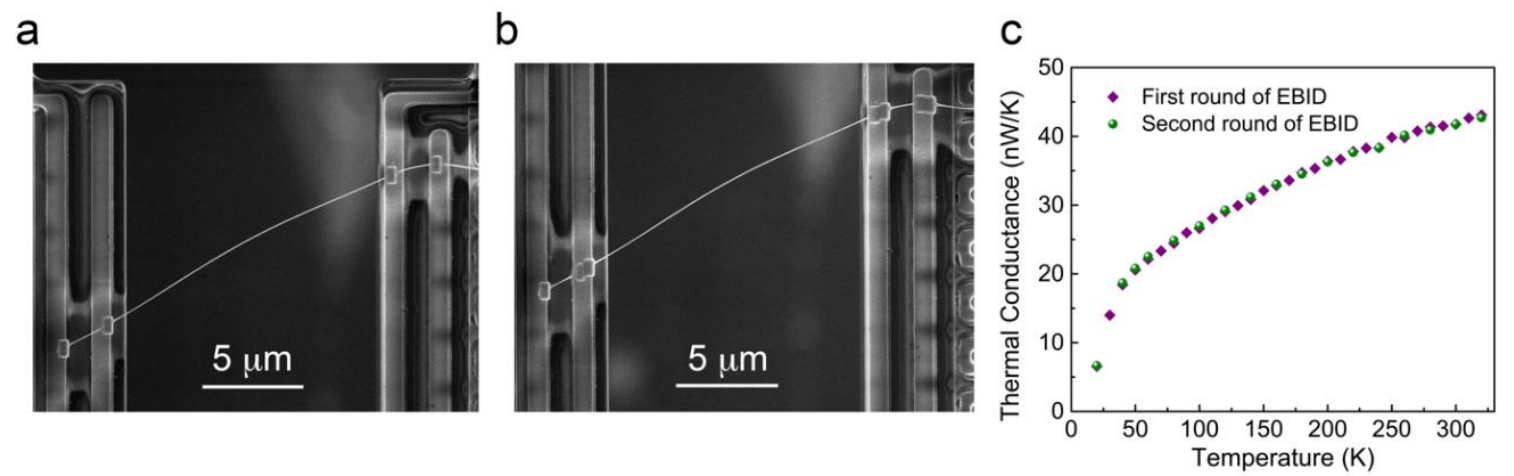

Figure S3. SEM images of a single Ag nanowire after (a) the first and (b) second rounds of EBID. (c) Measured thermal conductance after the first and second rounds of EBID. The suspended length and the hydraulic diameter of the Ag nanowire is $15 \mu \mathrm{m}$ and $43 \mathrm{~nm}$, respectively.

\section{Bending effect}

During the sample preparation, we found that the local bending could be induced by probe manipulation. We experimentally examined whether the local bending alters the transport properties of an Ag nanowire. Specifically, after thermal and electrical measurements of an Ag nanowire, an artificial kink was introduced in the middle of the wire, as shown in Fig. S4b, using a sharp probe. Then, we repeated the thermal and electrical properties measurements and compared the results before and after introducing the local bending. As shown in Figs. S4c 
and S4d, the measurement results with and without the kink essentially overlap with each other, indicating that local bending has negligible effect on the transport properties of the $\mathrm{Ag}$ nanowire.
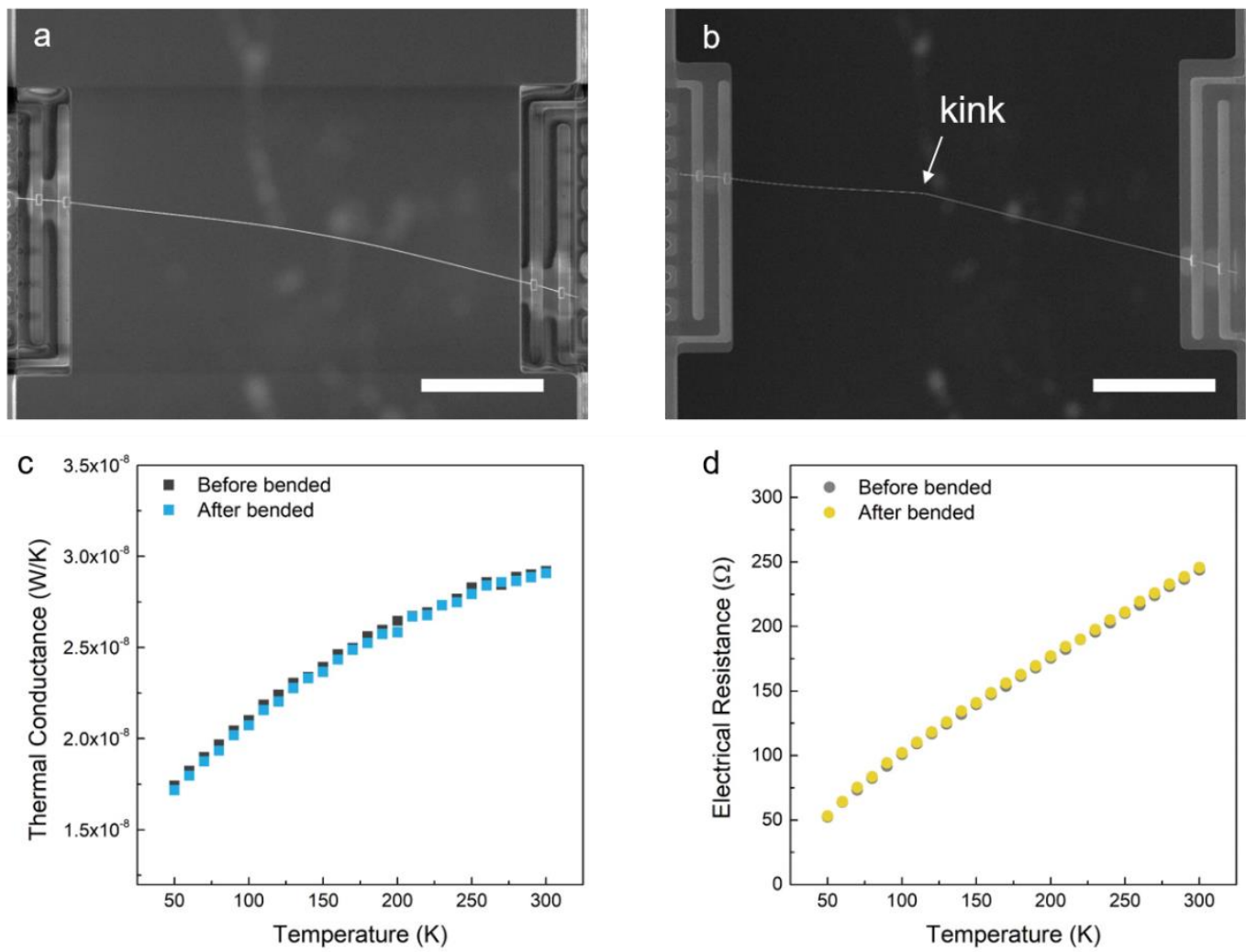

Figure S4. SEM images of an Ag nanowire (a) before and (b) after introducing an artificial kink. The hydraulic diameter of the Ag nanowire is $65 \mathrm{~nm}$. The scale bar is $10 \mu \mathrm{m}$. (c) Thermal conductance and (d) electrical resistance of the Ag nanowire with and without the kink.

\section{Fitting of electrical conductivity}

The temperature-dependent electrical conductivity (the reciprocal of electrical resistivity) of the Ag nanowire is fitted by combining the Bloch-Grüneisen formula with the Fuchs-Sondheimer reduction function ${ }^{6-9}$ to account for the effects of boundary scattering and surface specularity on electron transport in the Ag nanowire. The electrical resistivity of bulk $\operatorname{Ag}\left(\rho_{\text {bulk }}\right)$ is well described by the Bloch-Grüneisen formula ${ }^{6}$ 


$$
\rho_{\text {bulk }}(T)=\rho_{0}+\rho_{\text {e-ph }}(T)=\rho_{0}+\alpha_{\text {e-ph }}\left(\frac{T}{\theta_{\mathrm{D}}}\right)^{5} \int_{0}^{\theta_{\mathrm{D}} / T} \frac{x^{5} e^{x}}{\left(e^{x}-1\right)^{2}} d x
$$

where ${ }_{0}$ is the residual resistivity arising from structural imperfection scattering that is temperature independent. $\quad$ e-ph represents the resistivity due to electron-phonon scattering. $\alpha_{\mathrm{e}-\mathrm{ph}}$ and $\theta_{\mathrm{D}}$ are the electron-phonon coupling parameter and the Debye temperature, respectively. For the Ag nanowire, boundary scattering at the nanowire surface also contributes to the electrical resistivity as the electron MFP is comparable to the nanowire size. To explicitly reflect the surface contribution, we calculate a reduction function following the FuchsSondheimer approach, which relates the nanowire resistivity with the bulk value as ${ }^{7,8}$

$$
\begin{aligned}
& \left(\frac{\rho_{\text {bulk }}}{\rho_{\text {nw }}}\right)_{0, \lambda_{\mathrm{e}}}=\frac{3}{2 \pi r_{0}^{2}} \int_{0}^{r_{0}} r d r \int_{0}^{2 \pi} d \varphi \int_{0}^{\pi} \sin \theta \cos ^{2} \theta\left(1-e^{-\frac{\sqrt{r^{2} \cos ^{2} \varphi+r_{0}^{2}-r^{2}}-r \cos \varphi}{\lambda_{\mathrm{e}} \sin \theta}}\right) d \theta, \\
& \left(\frac{\rho_{\text {bulk }}}{\rho_{\mathrm{nw}}}\right)_{p, \lambda_{\mathrm{e}}}=(1-p)^{2} \sum_{m=1}^{\infty}\left[m p^{m-1}\left(\frac{\rho_{\text {bulk }}}{\rho_{\mathrm{nw}}}\right)_{0, \frac{\lambda_{\mathrm{e}}}{m}}\right],
\end{aligned}
$$

where ${ }_{\mathrm{nw}}$ is the electrical resistivity of the $\mathrm{Ag}$ nanowire, $r_{0}$ is the wire radius, is the polar angle between the electron traveling direction and the wire axial direction, is the azimuthal angle, $\lambda_{\mathrm{e}}$ is the electron MFP in bulk Ag, and $p$ is the specularity parameter. The best fitting curve is obtained with $\rho_{0}=1.13 \times 10^{-11} \Omega \cdot \mathrm{m}, \alpha_{\mathrm{e}-\mathrm{ph}}=5.14 \times 10^{-8} \Omega \cdot \mathrm{m}, \theta_{\mathrm{D}}=230 \mathrm{~K}$, and $p=0.64$, which matches the measured electrical conductivity very well as shown in Fig. 2c. 


\section{Electron MFP}

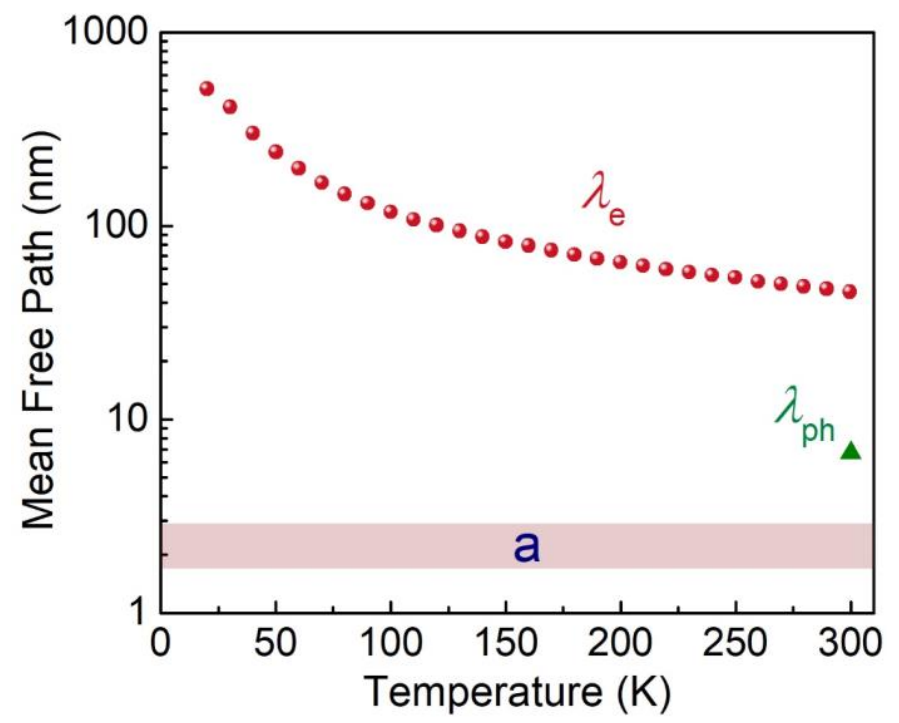

Figure S5. Electron MFP $\left(\lambda_{\mathrm{e}}\right)$ estimated from the measured electrical conductivity of the Ag nanowire using the Drude's relation. The phonon MFP $\left(\lambda_{\text {ph }}\right)$ predicted for Ag at $300 \mathrm{~K}$ by first principles calculations ${ }^{10}$ and the range of the extracted contact size (a) are also shown for comparison. 


\section{Additional experimental results}

a

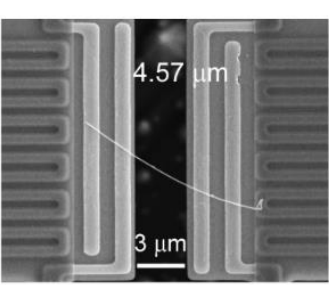

d

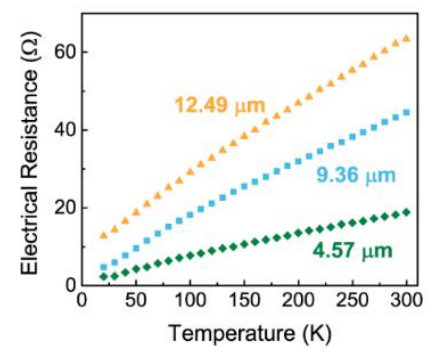

b
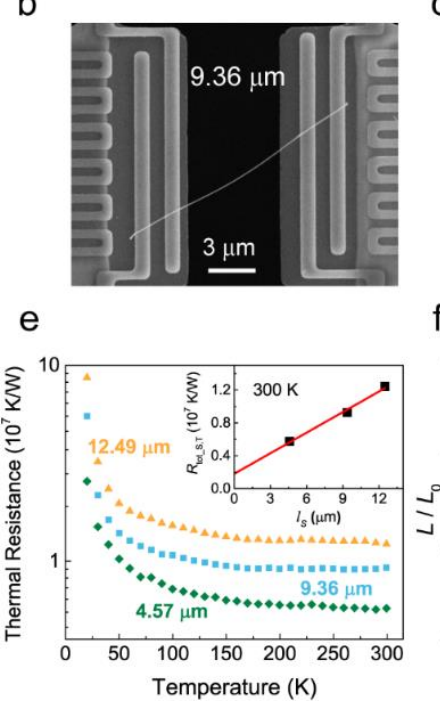

C

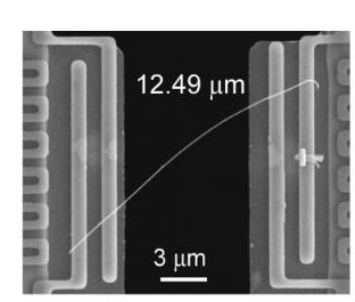

$\mathrm{f}$

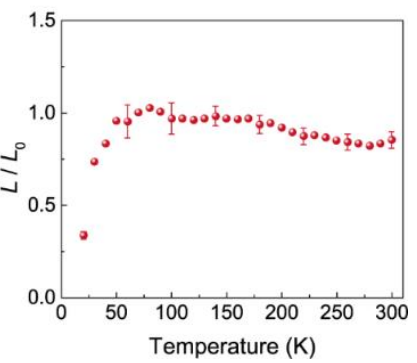

Figure S6. (a-c) SEM images of three single Ag nanowires with different suspended lengths for S2. (d) Measured electrical resistance. (e) Measured thermal resistance. The inset shows the linear fitting of the measured thermal resistance $\left(R_{\mathrm{tot} \_\mathrm{S}, \mathrm{T}}\right)$ as a function of the suspended length $\left(l_{\mathrm{S}}\right)$ at $300 \mathrm{~K}$. (f) Derived $L / L_{0}$ of the single Ag nanowire for S2. The hydraulic diameter of $\mathrm{S} 2$ is $72 \mathrm{~nm}$. 
a

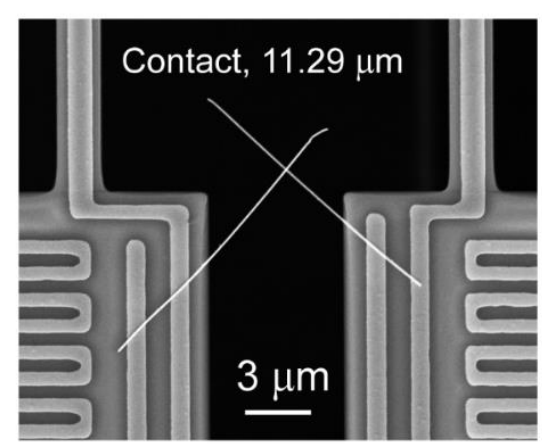

C

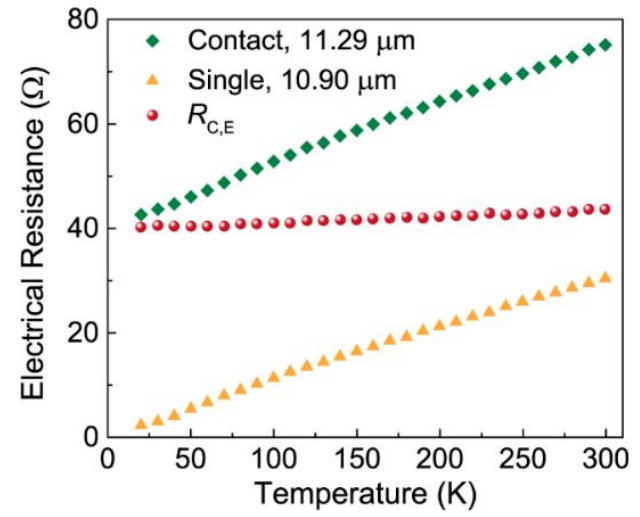

b

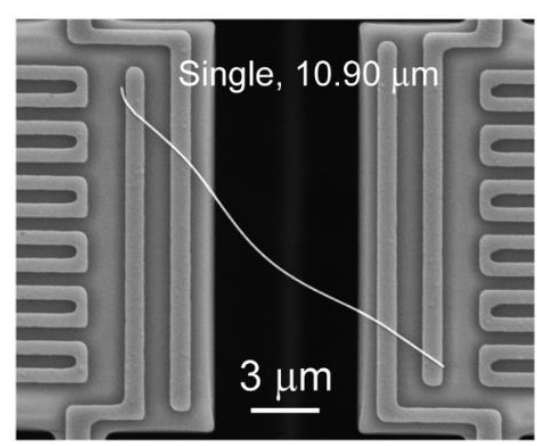

d

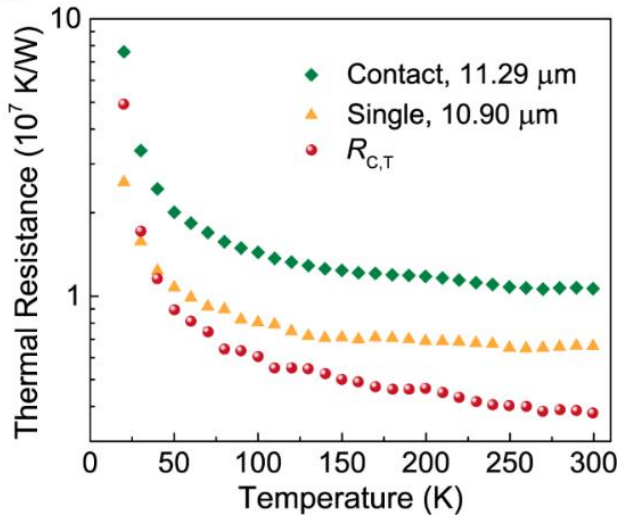

Figure S7. SEM images of (a) two Ag nanowires with a point contact and (b) a single Ag nanowire for S3. (c) Measured electrical resistance of the point-contact sample and the single Ag nanowire and the extracted electrical resistance of the point contact. (d) Measured thermal resistance of the point-contact sample and the single Ag nanowire and the extracted thermal resistance of the point contact. The hydraulic diameter of S3 is $91 \mathrm{~nm}$. 
a

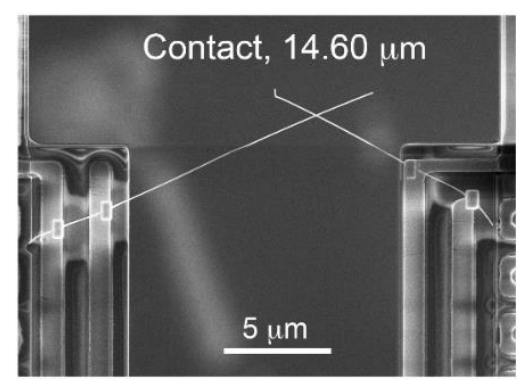

C

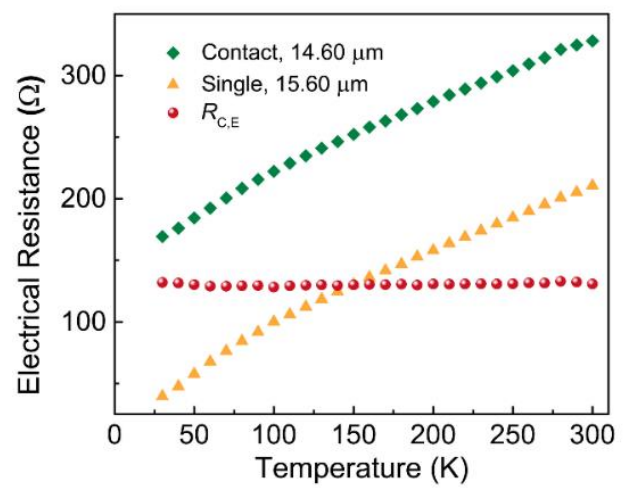

b

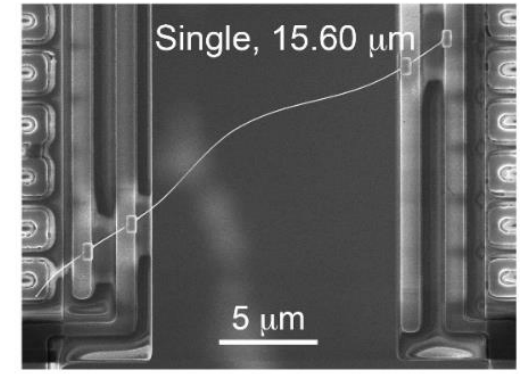

d

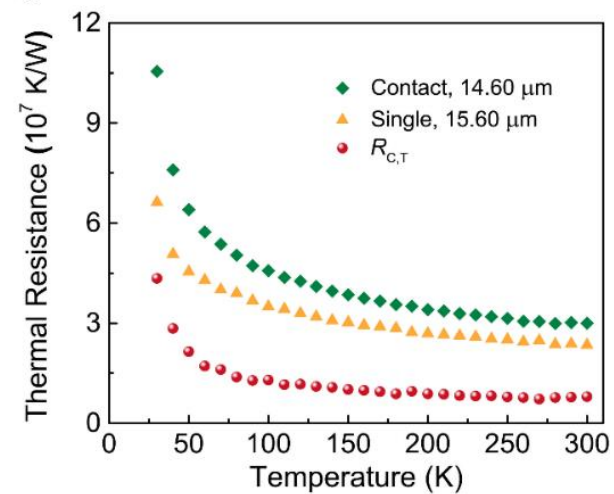

Figure S8. SEM images of (a) two Ag nanowires with a point contact and (b) a single Ag nanowire for S4. The EBID treatment was conducted to enhance the contact between the nanowire and the Pt electrodes. (c) Measured electrical resistance of the point-contact sample and the single Ag nanowire and the extracted electrical resistance of the point contact. (d) Measured thermal resistance of the point-contact sample and the single Ag nanowire and the extracted thermal resistance of the point contact. The hydraulic diameter of S4 is $43 \mathrm{~nm}$.

\section{Sharvin resistance}

The expression of Sharvin resistance is derived for a contact between two objects with sizes larger than the carrier MFP, as shown in Fig. S9a below. For the measured smallest wire with a diameter of $43 \mathrm{~nm}$, the cap of the hemisphere with the carrier MFP as the radius is truncated by the nanowire surface, as shown in Fig. S9b. In this case, it seems that no electron will be emitted from the truncated region and point to the contact, and the formula for Sharvin resistance needs to be modified. However, since the boundary is a free surface, the electrons emitted upwards from the mirror image region of the truncated cap in the nanowire will be 
reflected from the nanowire surface. Assuming that electrons transport isotropically, the reflected electron flux should be equivalent to that emitted from the cap region if it is not truncated. In view of this, the expression of Sharvin resistance should not be altered significantly. We believe that our thought is conceptually correct but detailed mathematical derivation could be quite involving, especially for thermal resistance, unless we also make the assumption that the temperature inside the hemisphere is uniform, which might not be exactly the case in the nano-confined regime. In addition, reflection of electrons from the free surface could also lead to additional complication. ${ }^{5}$ While further quantitative theoretical analysis has to be conducted to examine this size effect on Sharvin resistance, we believe that the modification should not be significant based on the above physical picture.

a

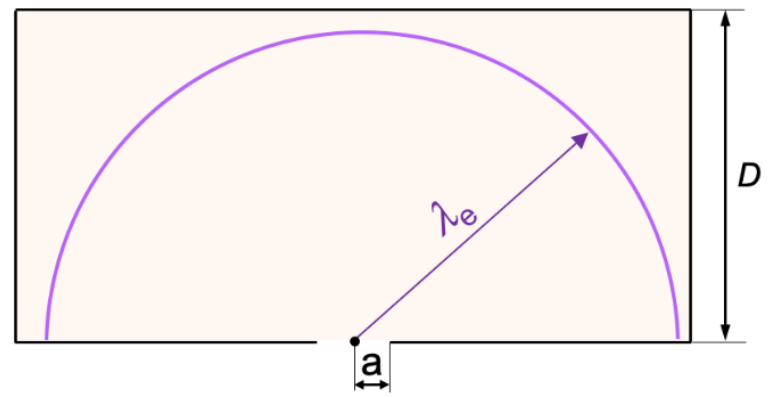

b

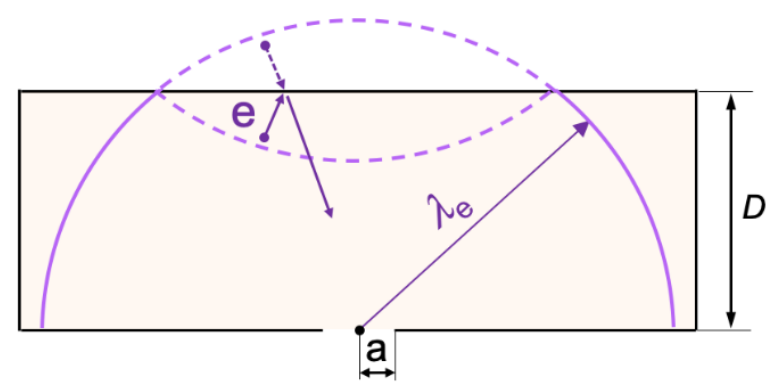

Figure S9. Schematics for deriving the ballistic contact resistance. (a) The wire diameter $(D)$ is larger than the electron $\operatorname{MFP}\left(\lambda_{\mathrm{e}}\right)$. (b) $D<\lambda_{\mathrm{e}}$.

\section{Contact area between Ag nanowires}

In a previous study, ${ }^{9}$ we have managed to experimentally measure the contact area between two Ag nanowires by first depositing the $\mathrm{Pt} / \mathrm{C}$ composite at the point contact, cutting through the point contact using a focused ion beam, and then etching Ag nanowires away. The contact area can be determined from the tilted SEM image as shown in Fig. S10 for the point contact between two Ag nanowires with a hydraulic diameter of $65 \mathrm{~nm}$. Seven measurements using the ImageJ software yield a contact area of $107.0 \pm 17.6 \mathrm{~nm}^{2}$, where the error stands for 
the standard deviation. This contact-area range corresponds to a contact radius of 5.3-6.3 nm. However, this measurement is extremely challenging, and we could only obtain such an image for one sample. Moreover, the approach also involves significant uncertainty as it can be imagined that in the close vicinity of the direct contact region where the gap between the two nanowires is only $<1$ nanometer apart, there might be no $\mathrm{Pt} / \mathrm{C}$ deposition as it is likely that the decomposed Pt/C gas atoms cannot reach there. As a result, the approach would yield a value larger than the actual contact area.

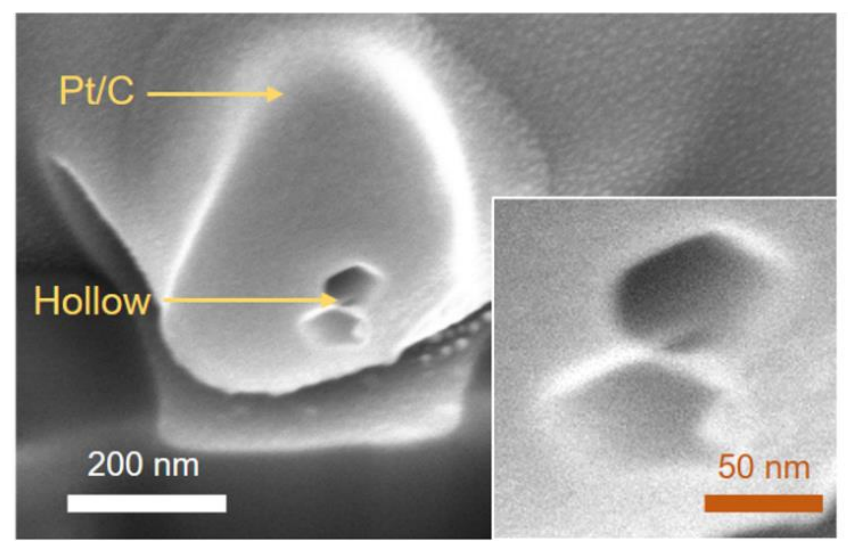

Figure S10. SEM images for determining the contact area between two Ag nanowires with a hydraulic diameter of $65 \mathrm{~nm}$.

\section{Uncertainty analysis}

The electrical resistance was measured via a four-probe method. The voltage and current were measured by using a low-noise voltage preamplifier (SR560, Stanford Research Systems) and a low-noise current preamplifier (SR570, Stanford Research Systems), respectively. The electrical conductivity of the Ag nanowire is calculated from the measured electrical resistance by

$$
\sigma=\frac{4 l_{\mathrm{S}}}{\pi D_{\mathrm{h}}^{2} R_{\mathrm{tot} \_\mathrm{S}, \mathrm{E}}} .
$$

The relative uncertainty in $\sigma$ can be estimated via the uncertainty propagation approach, ${ }^{11}$ 


$$
\frac{\delta \sigma}{\sigma}=\sqrt{4\left(\frac{\delta D_{\mathrm{h}}}{D_{\mathrm{h}}}\right)^{2}+\left(\frac{\delta l_{\mathrm{S}}}{l_{\mathrm{S}}}\right)^{2}+\left(\frac{\delta R_{\mathrm{tot} \_\mathrm{S}, \mathrm{E}}}{R_{\mathrm{tot} \_\mathrm{S}, \mathrm{E}}}\right)^{2}} .
$$

The uncertainty in $D_{\mathrm{h}}$ is estimated to be $5 \mathrm{~nm}$. The suspended length was measured from the SEM images, and the error $l_{\mathrm{S}}$ was estimated to be $200 \mathrm{~nm}$. The relative uncertainty in the measured electrical resistance is estimated to be $1 \%$.

The intrinsic thermal conductivity of the $\mathrm{Ag}$ nanowire is extracted by the linear leastsquares fitting of the measured thermal resistance as a function of the suspended length for the three single Ag nanowires. The intrinsic thermal resistance per unit length for the Ag nanowire $\left(R_{\mathrm{Ag}, \mathrm{T}}^{\prime}\right)$ and $R_{\mathrm{WM}, \mathrm{T}}$ can be expressed as

$$
\begin{aligned}
& R_{\mathrm{Ag}, \mathrm{T}}^{\prime}=\frac{n \sum_{i=1}^{n} l_{\mathrm{S}, i} R_{\mathrm{tot} \_\mathrm{S}, \mathrm{T}_{-} i}-\sum_{i=1}^{n} l_{\mathrm{S}, i} \sum_{i=1}^{n} R_{\mathrm{tot} \_\mathrm{S}, \mathrm{T}_{-} i}}{n \sum_{i=1}^{n}\left(l_{\mathrm{S}, i}^{2}\right)-\left(\sum_{i=1}^{n} l_{\mathrm{S}, i}\right)^{2}}, \\
& R_{\mathrm{WM}, \mathrm{T}}=\frac{\sum_{i=1}^{n}\left(l_{\mathrm{S}, i}^{2}\right) \sum_{i=1}^{n} R_{\mathrm{tot} \_\mathrm{S}, \mathrm{T}_{-} i}-\sum_{i=1}^{n} l_{\mathrm{S}, i} \sum_{i=1}^{n}\left(l_{\mathrm{S}, i} R_{\mathrm{tot} \_\mathrm{S}, \mathrm{T}_{-} i}\right)}{n \sum_{i=1}^{n}\left(l_{\mathrm{S}, i}^{2}\right)-\left(\sum_{i=1}^{n} l_{\mathrm{S}, i}\right)^{2}} .
\end{aligned}
$$

The relative uncertainty in $R_{\mathrm{Ag}, \mathrm{T}}^{\prime}$ and $R_{\mathrm{WM}, \mathrm{T}}$ can be estimated by

$$
\begin{aligned}
& \frac{\delta R_{\mathrm{Ag}, \mathrm{T}}^{\prime}}{R_{\mathrm{Ag}, \mathrm{T}}^{\prime}}=\sqrt{\sum_{i=1}^{n}\left(\frac{\partial R_{\mathrm{Ag}, \mathrm{T}}^{\prime}}{\partial R_{\mathrm{tot} \_\mathrm{S}, \mathrm{T}_{-} i}}\right)^{2}\left(\frac{\delta R_{\mathrm{tot} \_\mathrm{S}, \mathrm{T}_{-} i}}{R_{\mathrm{Ag}, \mathrm{T}}^{\prime}}\right)^{2}+\sum_{i=1}^{n}\left(\frac{\partial R_{\mathrm{Ag}, \mathrm{T}}^{\prime}}{\partial l_{\mathrm{S}, i}}\right)^{2}\left(\frac{\delta l_{\mathrm{S}, i}}{R_{\mathrm{Ag}, \mathrm{T}}^{\prime}}\right)^{2}}, \\
& \frac{\delta R_{\mathrm{WM}, \mathrm{T}}}{R_{\mathrm{WM}, \mathrm{T}}}=\sqrt{\sum_{i=1}^{n}\left(\frac{\partial R_{\mathrm{WM}, \mathrm{T}}}{\partial R_{\mathrm{tot} \_\mathrm{S}, \mathrm{T}_{-} i}}\right)^{2}\left(\frac{\delta R_{\mathrm{tot} \_\mathrm{S}, \mathrm{T}_{-} i}}{R_{\mathrm{WM}, \mathrm{T}}}\right)^{2}+\sum_{i=1}^{n}\left(\frac{\partial R_{\mathrm{WM}, \mathrm{T}}}{\partial l_{\mathrm{S}, i}}\right)^{2}\left(\frac{\delta l_{\mathrm{S}, i}}{R_{\mathrm{WM}, \mathrm{T}}}\right)^{2}},
\end{aligned}
$$

where $R_{\text {tot_S,T_i } i}$ and $l_{\mathrm{S}, i}$ are errors in the measured thermal resistance and the suspended length, respectively. The relative uncertainty in the measured thermal resistance is evaluated 
by using the Monte Carlo method, which is less than $4 \%$ in the temperature range of $50-100 \mathrm{~K}$ and $\sim 2 \%$ above $100 \mathrm{~K}$. The relative uncertainty in $\kappa$ can be estimated by

$$
\frac{\delta \kappa}{\kappa}=\sqrt{\left(\frac{\delta R_{\mathrm{Ag}, \mathrm{T}}^{\prime}}{R_{\mathrm{Ag}, \mathrm{T}}^{\prime}}\right)^{2}+4\left(\frac{\delta D_{\mathrm{h}}}{D_{\mathrm{h}}}\right)^{2}} .
$$

The normalized effective Lorenz number of the single Ag nanowire is calculated by

$$
\frac{L}{L_{0}}=\frac{\kappa}{\sigma T L_{0}}=\frac{R_{\mathrm{Ag}, \mathrm{E}}^{\prime}}{R_{\mathrm{Ag}, \mathrm{T}}^{\prime} T L_{0}} .
$$

Note that the cross-sectional area of the Ag nanowire is cancelled out when calculating $L / L_{0}$.

The relative uncertainty in $L / L_{0}$ is

$$
\frac{\left(L / L_{0}\right)}{L / L_{0}}=\sqrt{\left(\frac{R_{\mathrm{Ag}, \mathrm{T}}}{R_{\mathrm{Ag}, \mathrm{T}}}\right)^{2}+\left(\frac{R_{\mathrm{Ag}, \mathrm{E}}^{\prime}}{R_{\mathrm{Ag}, \mathrm{E}}}\right)^{2}+\left(\frac{T}{T}\right)^{2}},
$$

where $R_{\mathrm{Ag}, \mathrm{E}}^{\prime}$ and $\delta R_{\mathrm{Ag}, \mathrm{E}}^{\prime}$ are the measured electrical ressitance per unit length for the $\mathrm{Ag}$ nanowire and its uncertainty. Temperautre was measured by using a type E thermocouple and its uncertainty was less than $0.4 \%$. The estimated relative uncertainty in $L / L_{0}$ of the single $\mathrm{Ag}$ nanowire is less than $9 \%$ and $6 \%$ for S1 and S2, respectively, above $100 \mathrm{~K}$.

For $\mathrm{S} 1$, the electrical $\left(R_{\mathrm{C}, \mathrm{E}}\right)$ and thermal resistance $\left(R_{\mathrm{C}, \mathrm{T}}\right)$ of the point contact can be determined from Eq. (S2). The relative uncertainty in $R_{\mathrm{C}, \mathrm{E}}$ and $R_{\mathrm{C}, \mathrm{T}}$ is

$$
\begin{aligned}
& \frac{R_{\mathrm{C}, \mathrm{E}}}{R_{\mathrm{C}, \mathrm{E}}}=\sqrt{\left(\frac{R_{\mathrm{tot} C \mathrm{C}, \mathrm{E}}}{R_{\mathrm{C}, \mathrm{E}}}\right)^{2}+\left(R_{\mathrm{Ag}, \mathrm{E}} \frac{l_{\mathrm{C}}}{R_{\mathrm{C}, \mathrm{E}}}\right)^{2}+\left(l_{\mathrm{C}} \frac{R_{\mathrm{Ag}, \mathrm{E}}}{R_{\mathrm{C}, \mathrm{E}}}\right)^{2}}, \\
& \frac{R_{\mathrm{C}, \mathrm{T}}}{R_{\mathrm{C}, \mathrm{T}}}=\sqrt{\left(\frac{R_{\mathrm{tot} \_\mathrm{C}, \mathrm{T}}}{R_{\mathrm{C}, \mathrm{T}}}\right)^{2}+\left(\frac{R_{\mathrm{WM}, \mathrm{T}}}{R_{\mathrm{C}, \mathrm{T}}}\right)^{2}+\left(R_{\mathrm{Ag}, \mathrm{T}} \frac{l_{\mathrm{C}}}{R_{\mathrm{C}, \mathrm{T}}}\right)^{2}+\left(l_{\mathrm{C}} \frac{R_{\mathrm{Ag}, \mathrm{T}}}{R_{\mathrm{C}, \mathrm{T}}}\right)^{2}} .
\end{aligned}
$$

$L / L_{0}$ of the point contact is calculated by

$$
\frac{L}{L_{0}}=\frac{R_{\mathrm{C}, \mathrm{E}}}{R_{\mathrm{C}, \mathrm{T}} T L_{0}} .
$$


Therefore, the relative uncertainty in $L / L_{0}$ is

$$
\frac{\left(L / L_{0}\right)}{L / L_{0}}=\sqrt{\left(\frac{R_{\mathrm{C}, \mathrm{E}}}{R_{\mathrm{C}, \mathrm{E}}}\right)^{2}+\left(\frac{R_{\mathrm{C}, \mathrm{T}}}{R_{\mathrm{C}, \mathrm{T}}}\right)^{2}+\left(\frac{T}{T}\right)^{2}} .
$$

The relative uncertainty in $L / L_{0}$ of the point contact for S1 is estimated to be less than $11 \%$ above $100 \mathrm{~K}$.

For $\mathrm{S} 3$ and $\mathrm{S} 4, R_{\mathrm{C}, \mathrm{E}}$ and $R_{\mathrm{C}, \mathrm{T}}$ of the point contact were obtained by

$$
\begin{aligned}
& R_{\mathrm{C}, \mathrm{E}}=R_{\mathrm{tot} \_\mathrm{C}, \mathrm{E}}-R_{\mathrm{tot} \_\mathrm{S}, \mathrm{E}} \times l_{\mathrm{C}} / l_{\mathrm{S}}, \\
& R_{\mathrm{C}, \mathrm{T}}=R_{\mathrm{tot} \_\mathrm{C}, \mathrm{T}}-R_{\mathrm{tot} \_\mathrm{S}, \mathrm{T}} \times l_{\mathrm{C}} / l_{\mathrm{S}} .
\end{aligned}
$$

Since the contact electrical resistance between the nanowire and the Pt electrodes is negligible, the difference between $l_{\mathrm{C}}$ and $l_{\mathrm{S}}$ will not cause additional error in $R_{\mathrm{C}, \mathrm{E}}$. The relative uncertainty in $R_{\mathrm{C}, \mathrm{E}}$ can be estimated by

$$
\frac{R_{\mathrm{C}, \mathrm{E}}}{R_{\mathrm{C}, \mathrm{E}}}=\sqrt{\left(\frac{R_{\mathrm{tot} \_\mathrm{C}, \mathrm{E}}}{R_{\mathrm{C}, \mathrm{E}}}\right)^{2}+\left(\frac{l_{\mathrm{C}}}{l_{\mathrm{S}}}\right)^{2}\left(\frac{R_{\mathrm{tot} \_\mathrm{S}, \mathrm{E}}}{R_{\mathrm{C}, \mathrm{E}}}\right)^{2}+\left(\frac{R_{\mathrm{tot} \mathrm{S}, \mathrm{E}}}{l_{\mathrm{S}}}\right)^{2}\left(\frac{l_{\mathrm{C}}}{R_{\mathrm{C}, \mathrm{E}}}\right)^{2}+\left(\frac{\left.R_{\mathrm{tot} \mathrm{S}, \mathrm{E} \mathrm{C}}\right)}{l_{\mathrm{S}}^{2}}\right)^{2}\left(\frac{l_{\mathrm{S}}}{R_{\mathrm{C}, \mathrm{E}}}\right)^{2}} .
$$

However, the non-negligible $R_{\mathrm{WM}, \mathrm{T}}$ and the difference between $l_{\mathrm{C}}$ and $l_{\mathrm{S}}$ will induce an error in $R_{\mathrm{C}, \mathrm{T}}$,

$$
E r r=\left|R_{\mathrm{wM}, \mathrm{T}} \times\left(l_{\mathrm{C}}-l_{\mathrm{S}}\right) / l_{\mathrm{S}}\right|
$$

The relative uncertainty in $R_{\mathrm{C}, \mathrm{T}}$ is expressed as

$$
\frac{R_{\mathrm{C}, \mathrm{T}}}{R_{\mathrm{C}, \mathrm{T}}}=\sqrt{\left(\frac{R_{\mathrm{tot} \mathrm{C}, \mathrm{T}}}{R_{\mathrm{C}, \mathrm{T}}}\right)^{2}+\left(\frac{l_{\mathrm{C}}}{l_{\mathrm{S}}}\right)^{2}\left(\frac{R_{\mathrm{tot} \_\mathrm{S}, \mathrm{T}}}{R_{\mathrm{C}, \mathrm{T}}}\right)^{2}+\left(\frac{R_{\mathrm{tot} S \mathrm{~S}, \mathrm{~T}}}{l_{\mathrm{S}}}\right)^{2}\left(\frac{l_{\mathrm{C}}}{R_{\mathrm{C}, \mathrm{T}}}\right)^{2}+\left(\frac{R_{\mathrm{tot} \mathrm{S}, \mathrm{T} \mathrm{T}} l_{\mathrm{C}}}{l_{\mathrm{S}}^{2}}\right)^{2}\left(\frac{l_{\mathrm{S}}}{R_{\mathrm{C}, \mathrm{T}}}\right)^{2}+\left(\frac{E r r}{R_{\mathrm{C}, \mathrm{T}}}\right)^{2}} .
$$

For S1 and S3, the contact between the Ag nanowire and the Pt electrodes was treated by current-induced welding. Given their nearly identical hydraulic diameters $(92 \mathrm{~nm}$ and $91 \mathrm{~nm}$ for $\mathrm{S} 1$ and $\mathrm{S} 3$, respectively), it is reasonable to use $R_{\mathrm{WM}, \mathrm{T}}$ extracted for $\mathrm{S} 1$ to estimate $R_{\mathrm{WM}, \mathrm{T}}$ for S3. For S4, the contact between the Ag nanowire and the Pt electrodes was treated by alcohol evaporation and then EBID of Pt/C. As mentioned above, $R_{\mathrm{WM}, \mathrm{T}}$ is negligible after the 
EBID treatment. The estimated relative uncertainty in $L / L_{0}$ is less than $9 \%$ and $13 \%$ for $\mathrm{S} 3$ and S4, respectively, above $100 \mathrm{~K}$.

\section{Ratio of the Phonon to Electron Thermal Conductance}

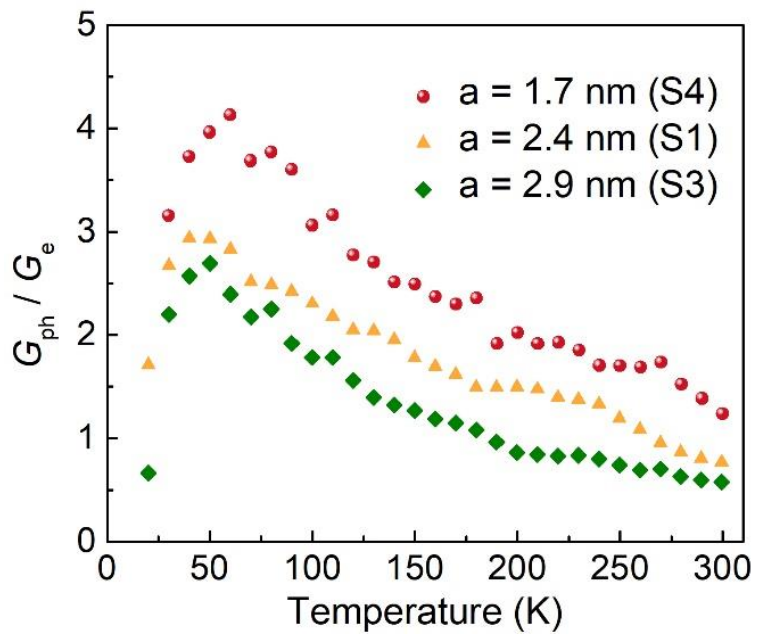

Figure S11. Ratio of the phonon to electron thermal conductance $\left(G_{\mathrm{ph}} / G_{\mathrm{e}}\right)$ of the point contact for three samples (S1, S3, and S4).

\section{Current-induced welding at the point contact}

a

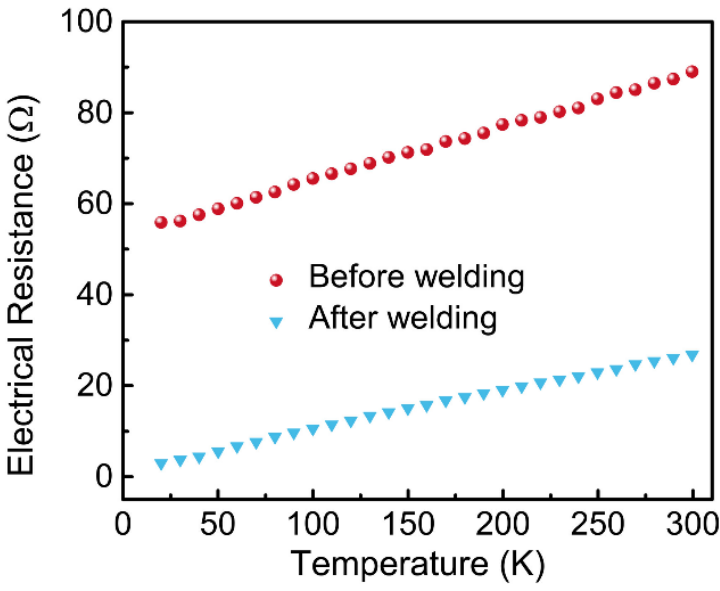

b

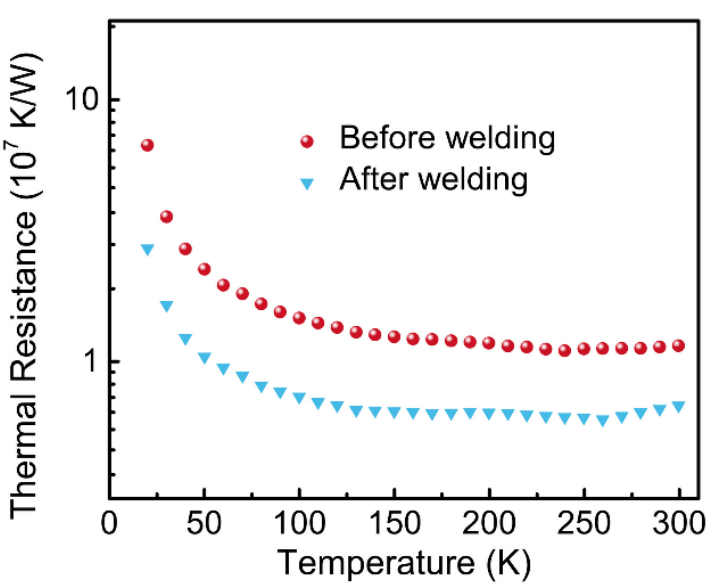

Figure S12. (a) Measured electrical and (b) thermal resistance of the contact sample (S1) before and after welding. 


\section{REFERENCES}

1. Shi, L.; Li, D.; Yu, C.; Jang, W.; Kim, D.; Yao, Z.; Kim, P.; Majumdar, A. Measuring thermal and thermoelectric properties of one-dimensional nanostructures using a microfabricated device. J. Heat Transfer 2003, 125, 881-888.

2. Wingert, M. C.; Chen, Z. C. Y.; Kwon, S.; Xiang, J.; Chen, R. Ultra-sensitive thermal conductance measurement of one-dimensional nanostructures enhanced by differential bridge. Rev. Sci. Instrum. 2012, 83, 024901.

3. Song, T. B.; Chen, Y.; Chung, C. H.; Yang, Y.; Bob, B.; Duan, H. S.; Li, G.; Tu, K. N.; Huang, Y.; Yang, Y. Nanoscale Joule heating and electromigration enhanced ripening of silver nanowire contacts. ACS Nano 2014, 8, 2804-2811.

4. Bellew, A. T.; Manning, H. G.; da Rocha, C. G.; Ferreira, M. S.; Boland, J. J. Resistance of single Ag nanowire junctions and their role in the conductivity of nanowire networks. ACS Nano 2015, 9, 11422-11429.

5. $\quad$ Yang, J.; Shen, M.; Yang, Y.; Evans, W. J.; Wei, Z.; Chen, W.; Zinn, A. A.; Chen, Y.; Prasher, R.; Xu, T. T.; Keblinski, P.; Li, D. Phonon transport through point contacts between graphitic nanomaterials. Phys. Rev. Lett. 2014, 112, 205901.

6. Ziman, J. M. Electrons and Phonons: The Theory of Transport Phenomena in Solids; Oxford University Press: New York, 2001.

7. Chambers, R. G. The conductivity of thin wires in a magnetic field. Proceedings of the Royal Society of London. Series A, Mathematical and Physical Sciences 1950, 202, 378-394.

8. Steinhögl, W.; Schindler, G.; Steinlesberger, G.; Engelhardt, M. Size-dependent resistivity of metallic wires in the mesoscopic range. Phys. Rev. B 2002, 66, 075414.

9. Zhao, Y.; Fitzgerald, M. L.; Tao, Y.; Pan, Z.; Sauti, G.; Xu, D.; Xu, Y.-Q.; Li, D. Electrical and thermal transport through silver nanowires and their contacts: Effects of elastic stiffening. Nano Lett. 2020, 20, 7389-7396.

10. Tong, Z.; Li, S. H.; Ruan, X. L.; Bao, H. Comprehensive first-principles analysis of phonon thermal conductivity and electron-phonon coupling in different metals. Phys. Rev. B 2019, 100, 144306.

11. Coleman, H. W.; Steele, W. G. Experimentation, Validation, and Uncertainty Analysis for Engineers, 3rd ed.; John Wiley \& Sons, Inc.: Hoboken, NJ, 2009. 